European Journal of Contraception and Reproductive Health CareVolume 23, Issue 3, 4 May 2018, Pages 207-217

\title{
Uterine or paracervical lidocaine application for pain control during intrauterine contraceptive device insertion: a meta-analysis of randomised controlled trials(Review)
}

- Perez-Lopez, F.R.a, Email Author,

- Martinez-Dominguez, S.J.b,

- Perez-Roncero, G.R. ${ }^{\text {b }}$

- Hernandez, A.V.c,d

- View Correspondence (jump link)

- aDepartment of Obstetrics and Gynecology, University of Zaragoza Faculty of Medicine, Hospital Clinico Lozano-Blesa, Zaragoza, Spain

- $\quad{ }^{b} R e d$ de Investigacion de Ginecologia, Obstetricia y Reproduccion, , Instituto Aragones de Ciencias de la Salud (IACS), Zaragoza, Spain

- UUniversity of Conneticut/Hartford Hospital Evidence-based Practice Center, Hartford, CT, United States

View additional affiliations

Abstract_View references (35)

Objective: Systematic review and meta-analysis to assess the effects of uterine or paracervical lidocaine application on pain control during IUD insertion.

Methods: PubMed and five other electronic research databases were searched through 15 November 2017 for RCTs comparing lidocaine treatment vs. a control (placebo or no-intervention) to prevent pain during IUD insertion.

Searched terms included 'IUD insertion', 'lidocaine' and 'randomised controlled trial'. RCTs evaluating lidocaine treatment before IUD insertion without restriction of language, age and IUD type. Pain measured by visual pain scales at tenaculum placement, IUD insertion and immediate post-IUD insertion. Results of random effects meta-analyses were reported as mean differences (MDs) of visual pain scale (VPS) scores and their 95\% confidence intervals (Cls). Results: Eleven RCTs ( $n=1458$ women) reporting paracervical lidocaine block or uterine mucosa lidocaine application before IUD insertion. Lidocaine produced lower VPS scores during tenaculum placement (MD $-0.99,95 \% \mathrm{Cl}$ : -1.73 to -0.26 ), IUD insertion (MD $-1.26,95 \% \mathrm{Cl}$ : -2.23 to -0.29 ) and immediate post-IUD insertion period (MD $-1.25,95 \% \mathrm{Cl}:-2.17$ to -0.33 ). Conclusion: Lidocaine treatment was associated with modest reduction of pain during tenaculum placement and after IUD insertion. (C) 2018, (C) 2018 The European Society of Contraception and Reproductive Health.

SciVal Topic Prominence

Topic: Contraception | Contraceptive Agents | reversible contraceptive Prominence percentile: 98.886 
Reaxys Database Information

国 View Compounds

Author keywords

Genital painintrauterine contraceptive devicelUD insertionlevonorgestrel

intrauterine systemlidocainepain visual scaleprilocaine

Indexed keywords

EMTREE drug terms: lidocainelidocaine plus prilocaineplacebo

EMTREE medical terms: analgesiacomparative effectivenesscontrolled studyendometriumfemalegenita comparisonmeta analysisparacervical blockpriority journalrandomized contro reviewvisual analog scale

Chemicals and CAS Registry Numbers:

lidocaine, 137-58-6, 24847-67-4, 56934-02-2, 73-78-9; lidocaine plus prilocaine, 101362-25-8

- ISSN: 13625187

- CODEN: ECRCF

- Source Type: Journal

- Original language: English

- DOI: $10.1080 / 13625187.2018 .1469124$

- Document Type: Review

- Publisher: Taylor and Francis Ltd 